\title{
CONTINUOUS-TIME FINITE ELEMENT ANALYSIS OF MULTIPHASE FLOW IN GROUNDWATER HYDROLOGY
}

\author{
Zhangxin Chen, Magne Espedal and Richard E. Ewing \\ APPLICATIONS OF MATHEMATICS, 1996, TO APPEAR
}

\begin{abstract}
A nonlinear differential system for describing an air-water system in groundwater hydrology is given. The system is written in a fractional flow formulation, i.e., in terms of a saturation and a global pressure. A continuous-time version of the finite element method is developed and analyzed for the approximation of the saturation and pressure. The saturation equation is treated by a Galerkin finite element method, while the pressure equation is treated by a mixed finite element method. The analysis is carried out first for the case where the capillary diffusion coefficient is assumed to be uniformly positive, and is then extended to a degenerate case where the diffusion coefficient can be zero. It is shown that error estimates of optimal order in the $L^{2}$-norm and almost optimal order in the $L^{\infty}$-norm can be obtained in the nondegenerate case. In the degenerate case we consider a regularization of the saturation equation by perturbing the diffusion coefficient. The norm of error estimates depends on the severity of the degeneracy in diffusivity, with almost optimal order convergence for non-severe degeneracy. Existence and uniqueness of the approximate solution is also proven.
\end{abstract}

\section{Introduction}

In this paper we develop and analyze a finite element procedure for solving the following flow equations for an air-water system in groundwater hydrology, $\alpha=a, w[1],[11],[26]$ :

$$
\begin{array}{ll}
\frac{\partial\left(\phi \rho_{\alpha} s_{\alpha}\right)}{\partial t}+\nabla \cdot\left(\rho_{\alpha} u_{\alpha}\right)=f_{\alpha}, & x \in \Omega, t>0, \\
u_{\alpha}=-\frac{k k_{r \alpha}}{\mu_{\alpha}}\left(\nabla p_{\alpha}-\rho_{\alpha} g\right), & x \in \Omega, t>0,
\end{array}
$$

where $\Omega \subset \Re^{d}, d \leq 3$ is a porous medium, $\phi$ and $k$ are the porosity and absolute permeability of the porous system, $\rho_{\alpha}, s_{\alpha}, p_{\alpha}, u_{\alpha}$, and $\mu_{\alpha}$ are the density, saturation, pressure, volumetric velocity, and viscosity of the $\alpha$-phase, $f_{\alpha}$ is the source/sink term, $k_{r \alpha}$ is the relative permeability of the $\alpha$-phase, and $g$ is the gravitational, downward-pointing, constant vector. The most commonly encountered boundary conditions for groundwater reservoir simulation are of first-type and second-type:

$$
\begin{array}{ll}
p_{\alpha}=p_{\alpha D}(x, t), & x \in \Gamma_{1}, t>0, \\
u_{\alpha} \cdot \nu=d_{\alpha}(x, t), & x \in \Gamma_{2}, t>0
\end{array}
$$

1991 Mathematics Subject Classification. Primary 65N30, 76S05.

Key words and phrases. mixed method, finite element, compressible flow, porous media, error estimate, air-water system.

Partly supported by the Department of Energy under contract DE-ACOS-840R21400. 
where $p_{\alpha D}$ and $d_{\alpha}$ are given functions, $\partial \Omega=\Gamma_{1} \cup \Gamma_{2}$ with $\Gamma_{1}$ and $\Gamma_{2}$ being disjoint, and $\nu$ is the outer unit normal to $\partial \Omega$.

In most previous work on the flow simulation in groundwater reservoirs the air-phase equation is eliminated by the assumption that the air-phase remains essentially at atmospheric pressure [21], [23]. This assumption is reasonable in most cases because the mobility of air is much larger than that of water, due to the viscosity difference between the two fluids. When the air-phase pressure is assumed constant, the air-phase mass balance equation is eliminated, and thus only the water-phase equation remains. Namely, the Richards equation is used to model the movement of water in groundwater reservoirs. However, it provides no information on the motion of air. If contaminant transport is the main concern and the contaminant can be transported in the air-phase, the air-phase needs to be included to determine the advective component of air-phase contaminant transport [5]. Furthermore, the dynamic interaction between the air and water phases is also important in vapor extraction systems. Hence in these cases the coupled system of nonlinear equations for the air-water system must be solved.

The purpose of this paper is to develop and analyze a finite element procedure for approximating the solution of the coupled system of nonlinear equations for the air-water system in groundwater hydrology. In the next section we derive a fractional flow formulation for equations (1.1)-(1.4). Namely, the flow equations and boundary conditions are written in terms of a saturation and a global pressure. The fractional flow approach is motivated by petroleum reservoir simulation [6], [14]. The main reason for this approach is that efficient numerical methods can be devised to take advantage of many physical properties inherent in the flow equations. It should be emphasized that petroleum reservoir simulation is very different from groundwater reservoir simulation. The flow of two incompressible fluids (e.g., water and oil) is usually considered in the former case, while the air phase is compressible in the latter case. Also, in petroleum reservoirs total flux type boundary conditions are conveniently imposed and often used, but in groundwater reservoirs boundary conditions are usually specified for each fluid as in (1.3) and (1.4) and can be very complicated [11]. It turns out that compressibility and various boundary conditions complicate error analyses. Indeed, as shown here, if optimality is to be preserved for the finite element method under consideration, the standard error argument just fails unless we work with higher order time-differentiated forms of mixed finite element error equations.

The weak forms of the pressure-saturation equations are defined in section three. Then in section four we introduce a finite element procedure for the saturation and pressure equations. The saturation equation is approximated by a finite element method, while the pressure equation is treated by a mixed finite element method. It is well known that the physical transport dominates the diffusive effects in incompressible flow. In the air-water system studied here, the transport again dominates the entire process. Hence it is important to obtain good approximate velocities. This motivates the use of the parabolic mixed method, as in [8], [12], and [15], in the computation of the pressure and the velocity. Also, due to its convection-dominated feature, more efficient approximate procedures should be used to solve the saturation equation. However, since this is the first time to carry out an analysis for the present problem, it is of some importance to establish that the standard finite element method for this model converges at an asymptotically optimal rate for smooth problems. Characteristic Petrov-Galerkin methods based on operator splitting [17], transport diffusion methods [27], and other characteristic based methods will be considered in 
forthcoming papers.

Asymptotical analyses for continuous-time finite element methods are carried out first for the case where the capillary diffusion coefficient is assumed to be uniformly positive, and then for a degenerate case where the diffusion coefficient vanishes for two values of saturation. It is shown that error estimates of optimal order in the $L^{2}$-norm and almost optimal order in the $L^{\infty}$-norm can be obtained for the former case; see section five. The analysis for the fully discrete version is more delicate than that for the semidiscrete version, and will appear elsewhere. Furthermore, our techniques for the treatment of the parabolic mixed finite element method are very different from those given in [12] and [15].

In the degenerate case we consider a regularization of the saturation equation by perturbing the diffusion coefficient to obtain a nondegenerate problem with smooth solutions. It is shown that the regularized solutions converge to the original solution as the perturbation parameter goes to zero with specific convergence rates given. The norm of error estimates depends on the severity of the degeneracy in diffusivity, with almost optimal order convergence for the degeneracy under consideration; see section six.

\section{A fractional flow formulation}

In addition to (1.1) and (1.2), we impose the customary property that the fluid fills the volume:

$$
s_{a}+s_{w}=1
$$

and define the capillary pressure function $p_{c}$ by

$$
p_{c}\left(s_{w}\right)=p_{a}-p_{w} .
$$

Introduce the phase mobilities

$$
\lambda_{\alpha}=k_{r \alpha} / \mu_{\alpha}, \quad \alpha=a, w
$$

and the total mobility

$$
\lambda=\lambda_{a}+\lambda_{w} .
$$

To devise our numerical method, as mentioned in the introduction we rewrite (1.1)-(1.4) in a pressure-saturation formulation. For this, define the global pressure [6] with $s=s_{w}$ :

$$
\begin{aligned}
p & =\frac{1}{2}\left(p_{a}+p_{w}\right)+\frac{1}{2} \int_{s_{c}}^{s} \frac{\lambda_{a}-\lambda_{w}}{\lambda} \frac{d p_{c}}{d \xi} d \xi \\
& =p_{w}+\int_{0}^{p_{c}(s)}\left(\frac{\lambda_{a}}{\lambda}\right)\left(p_{c}^{-1}(\xi)\right) d \xi,
\end{aligned}
$$

where $p_{c}\left(s_{c}\right)=0$. As usual, assume that $\rho_{\alpha}$ depends on $p$ [6]. Then we define the total velocity

$$
u=-k \lambda(\nabla p-G(s, p))
$$

where

$$
G(s, p)=\frac{\lambda_{a} \rho_{a}+\lambda_{w} \rho_{w}}{\lambda} g
$$


Now it can be easily seen that

$$
\begin{aligned}
& u_{w}=q_{w} u+k \lambda_{a} q_{w} \nabla p_{c}-k \lambda_{a} q_{w} \tilde{\rho}, \\
& u_{a}=q_{a} u-k \lambda_{w} q_{a} \nabla p_{c}+k \lambda_{w} q_{a} \tilde{\rho},
\end{aligned}
$$

where $q_{\alpha}=\lambda_{\alpha} / \lambda, \alpha=a, w$, and $\tilde{\rho}=\left(\rho_{a}-\rho_{w}\right) g$. Consequently,

$$
u=u_{a}+u_{w}
$$

Equations (1.1) and (1.2) can be manipulated using (2.1)-(2.6) to have the pressure equation

$$
\nabla \cdot u=-\frac{\partial \phi}{\partial t}-\sum_{\alpha=w}^{a} \frac{1}{\rho_{\alpha}}\left(\phi s_{\alpha} \frac{\partial \rho_{\alpha}}{\partial t}+u_{\alpha} \cdot \nabla \rho_{\alpha}-f_{\alpha}\right),
$$

and the saturation equation

$$
\begin{aligned}
\phi \frac{\partial s_{w}}{\partial t}+ & \nabla \cdot\left(q_{w} u+k \lambda_{a} q_{w}\left(\nabla p_{c}-\tilde{\rho}\right)\right) \\
& =-s_{w} \frac{\partial \phi}{\partial t}-\frac{1}{\rho_{w}}\left(\phi s_{w} \frac{\partial \rho_{w}}{\partial t}+u_{w} \cdot \nabla \rho_{w}-f_{w}\right) .
\end{aligned}
$$

The terms $u_{\alpha} \cdot \nabla \rho_{\alpha}, \alpha=a, w$ are effectively quadratic in the velocities, which are usually small in almost all of the domain [6], [26], and can be neglected. For the simplicity of error analysis, we do so below. Also, the water phase is usually assumed to be incompressible. Then (2.7) and (2.8) can be simplified. However, we emphasize that these assumptions can be easily removed with the techniques presented here. After introducing the following notation:

$$
\begin{array}{ll}
c(s, p)=\frac{\phi s}{\rho_{a}} \frac{d \rho_{a}}{d p}, & D(s)=-k \lambda_{a} q_{w} \frac{d p_{c}}{d s}, \\
f(p)=\frac{f_{a}}{\rho_{a}}+\frac{f_{w}}{\rho_{w}}, & a(s)=k \lambda, \\
\tilde{f}_{w}=\frac{f_{w}}{\rho_{w}}, & b(s, p)=-k \lambda_{a} q_{w} \tilde{\rho},
\end{array}
$$

equations (2.7) and (2.8) can be now written as

$$
\begin{aligned}
& c(s, p) \frac{\partial p}{\partial t}+\nabla \cdot u=f(p), \\
& u=-a(s)(\nabla p-G(s, p)), \\
& \phi \frac{\partial s}{\partial t}-\nabla \cdot\left(D(s) \nabla s-q_{w} u-b(s, p)\right)=\tilde{f}_{w}-s \frac{\partial \phi}{\partial t} .
\end{aligned}
$$

The boundary conditions for the pressure-saturation equations become

$$
\begin{array}{ll}
p=p_{D}(x, t), & x \in \Gamma_{1}, t>0, \\
u \cdot \nu=\tilde{d}(x, t), & x \in \Gamma_{2}, t>0, \\
s=s_{D}(x, t), & x \in \Gamma_{1}, t>0, \\
\left(D(s) \nabla s-q_{w} u-b(s, p)\right) \cdot \nu=-d_{w}(x, t), & x \in \Gamma_{2}, t>0,
\end{array}
$$


where $s_{D}$ and $p_{D}$ are the transforms of $p_{w D}$ and $p_{a D}$ by (2.2) and (2.3), and $\tilde{d}=d_{a}+d_{w}$.

The model is completed by specifying the initial conditions

$$
\begin{array}{ll}
p(x, 0)=p^{0}(x), & x \in \Omega, \\
s(x, 0)=s^{0}(x), & x \in \Omega .
\end{array}
$$

The later analysis in $\S 5$ and $\S 6$ is given under a number of assumptions. First, the solution is assumed smooth; i.e., the external source terms are smoothly distributed, the coefficients are smooth, the boundary and initial data satisfy the compatibility condition, and the domain has at least the regularity required for a standard elliptic problem to have $H^{2}(\Omega)$-regularity and more if error estimates of order greater than one are required. Second, the coefficients $a(s), \phi$, and $c(s, p)$ are assumed bounded below positively:

$$
\begin{aligned}
& 0<a_{*} \leq a(s) \leq a^{*}<\infty \\
& 0<\phi_{*} \leq \phi(x) \leq \phi^{*}<\infty \\
& 0<c_{*} \leq c(s, p) \leq c^{*}<\infty .
\end{aligned}
$$

Finally, the capillary diffusion coefficient $D(s)$ is assumed to satisfy

$$
0<D_{*} \leq D(s) \leq D^{*}<\infty .
$$

While the phase mobilities can be zero, the total mobility is always positive [26]. The assumptions (2.18)-(2.20) are physically reasonable. Although the reasonableness of the assumption (2.21) is discussed in [14], the diffusion coefficient $D(s)$ can be zero in reality. It is for this reason that section seven is devoted to consideration of the case where the solution is not required smooth and the assumption (2.21) is removed. As a final remark, we mention that for the case where point sources and sinks occur in a porous medium, an argument was given in [18] for the incompressible miscible displacement problem and can be extended to the present case.

\section{Weak formulations}

To handle the difficulty associated with the inhomogeneous Neumann boundary condition (2.13) in the analysis of the mixed finite element method, let $\bar{d}$ be such that $\bar{d} \cdot \nu=\tilde{d}$ and introduce the change of variable $u=\tilde{u}+\bar{d}$ in equations (2.9)-(2.11). Then the homogeneous Neumann boundary condition holds for $\tilde{u}$. Thus, without loss of generality, we assume that $\tilde{d} \equiv 0$. Let

$$
\begin{aligned}
& H(\operatorname{div}, \Omega)=\left\{v \in\left(L^{2}(\Omega)\right)^{d}: \nabla \cdot v \in L^{2}(\Omega), d=2,3\right\}, \\
& V=\left\{v \in H(\operatorname{div}, \Omega): v \cdot \nu=0 \text { on } \Gamma_{2}\right\}, \\
& M=\left\{w \in H^{1}(\Omega): w=0 \text { on } \Gamma_{1}\right\} .
\end{aligned}
$$

The weak form of $(2.9)-(2.11)$ on which the finite element procedure is based is given below. Let $J=(0, T](T>0)$ is the time interval of interest. The mixed formulation for the pressure is defined by seeking a pair of maps $\{u, p\}: J \rightarrow V \times L^{2}(\Omega)$ such that

$$
\begin{array}{ll}
(\alpha(s) u, v)-(\nabla \cdot v, p)=(G(s, p), v)-\left\langle p_{D}, v \cdot \nu\right\rangle_{\Gamma_{1}}, & \forall v \in V, \\
\left(c(s, p) \frac{\partial p}{\partial t}, \psi\right)+(\nabla \cdot u, \psi)=(f(p), \psi), & \forall \psi \in L^{2}(\Omega),
\end{array}
$$


where $\alpha(s)=a(s)^{-1}$, the inner products $(\cdot, \cdot)$ are to be interpreted to be in $L^{2}(\Omega)$ or $\left(L^{2}(\Omega)\right)^{d}$, as appropriate, and $\langle\cdot, \cdot\rangle_{\Gamma_{1}}$ denotes the duality between $H^{1 / 2}\left(\Gamma_{1}\right)$ and $H^{-1 / 2}\left(\Gamma_{1}\right)$. The weak form for the saturation $s: J \rightarrow M+s_{D}$ is given by

$$
\left(\phi \frac{\partial s}{\partial t}, v\right)+\left(D(s) \nabla s-q_{w}(s) u-b(s, p), \nabla v\right)=\left(\tilde{f}_{w}-s \frac{\partial \phi}{\partial t}, v\right)-\left\langle d_{w}, v\right\rangle_{\Gamma_{2}}, \quad \forall v \in M
$$

where the boundary condition (2.15) is used. Finally, to treat the nonzero initial conditions imposed on $s$ and $p$ in (2.16) and (2.17), we introduce the following transformations in (3.1) and (3.2):

$$
\begin{aligned}
& s(x, t)=\tilde{s}(x, t)+s^{0}(x), \\
& p(x, t)=\tilde{p}(x, t)+p^{0}(x), \\
& u(x, t)=\tilde{u}(x, t)+u^{0}(x),
\end{aligned}
$$

where $u^{0}=-a\left(s^{0}\right)\left(\nabla p^{0}-G\left(s^{0}, p^{0}\right)\right)$ and $\tilde{u}=-a\left(\tilde{s}+s^{0}\right)\left(\nabla\left(\tilde{p}+p^{0}\right)-G\left(\tilde{s}+s^{0}, \tilde{p}+p^{0}\right)\right)-u^{0}(x)$. Then we have zero initial conditions for $\tilde{s}, \tilde{p}$, and $\tilde{u}$. Thus, without loss of generality again, we assume that

$$
s^{0}=p^{0}=u^{0} \equiv 0
$$

The reason for introducing these transformations to have zero initial conditions is to validate equation (5.15) below.

\section{Finite element procedures}

Let $\Omega$ be a polygonal domain. For $0<h_{p}<1$ and $0<h<1$, let $T_{h_{p}}$ and $T_{h}$ be quasi-regular partitions into elements, say, simplexes, rectangular parallelepipeds, and/or prisms. In both partitions, we also need that adjacent elements completely share their common edge or face. Let $M_{h} \subset W^{1, \infty}(\Omega) \cap M$ be a standard $C^{0}$-finite element space associated with $T_{h}$ such that

$$
\inf _{\psi \in M_{h}}\|v-\psi\|_{1, q} \leq C\left(\sum_{K} h_{K}^{2 k}\|v\|_{k+1, q, K}^{2}\right)^{1 / 2}, \quad k \geq 1,1 \leq q \leq \infty
$$

where $h_{K}=\operatorname{diam}(K), K \in T_{h}$ and $\|v\|_{k, q, K}$ is the norm in the Sobolev space $W^{k, q}(K)$ (we omit $K$ when $k=\Omega$ and $\left.\|v\|_{k, K}=\|v\|_{k, 2, K}\right)$. Also, let $V_{h} \times W_{h}=V_{h_{p}} \times W_{h_{p}} \subset V \times L^{2}(\Omega)$ be the Raviart-Thomas-Nedelec [28], [24], the Brezzi-Douglas-Fortin-Marini [3], the BrezziDouglas-Marini [4] (if $d=2$ ), the Brezzi-Douglas-Durán-Fortin [2] (if $d=3$ ), or the Chen-Douglas [10] mixed finite element space associated with the partition $T_{h_{p}}$ of index such that the approximation properties below are satisfied:

$$
\begin{array}{ll}
\inf _{\psi \in V_{h}}\|v-\psi\| \leq C\left(\sum_{K} h_{p, K}^{2 r}\|v\|_{r, K}^{2}\right)^{1 / 2}, & 0 \leq r \leq k^{*}+1 \\
\inf _{\psi \in V_{h}}\|\nabla \cdot(v-\psi)\| \leq C\left(\sum_{K} h_{p, K}^{2 r}\|\nabla \cdot v\|_{r, K}^{2}\right)^{1 / 2}, & 0 \leq r \leq k^{* *} \\
\inf _{\psi \in W_{h}}\|w-\psi\| \leq C\left(\sum_{K} h_{p, K}^{2 r}\|w\|_{r, K}^{2}\right)^{1 / 2}, & 0 \leq r \leq k^{* *}
\end{array}
$$


where $h_{p, K}=\operatorname{diam}(K), K \in T_{h_{p}},\|v\|=\|v\|_{0}, k^{* *}=k^{*}+1$ for the first two spaces, $k^{* *}=k^{*}$ for the second two spaces, and both cases are included in the last space. We are now in a position to introduce our finite element procedure.

The continuous-time finite element method is given as follows. The approximation procedure for the pressure is defined by the mixed method for a pair of maps $\left\{u_{h}, p_{h}\right\}$ : $J \rightarrow V_{h} \times W_{h}$ such that

$$
\begin{array}{ll}
\left(\alpha\left(s_{h}\right) u_{h}, v\right)-\left(\nabla \cdot v, p_{h}\right)=\left(G\left(s_{h}, p_{h}\right), v\right)-\left\langle p_{D}, v \cdot \nu\right\rangle_{\Gamma_{1}}, & \forall v \in V_{h}, \\
\left(c\left(s_{h}, p_{h}\right) \frac{\partial p_{h}}{\partial t}, \psi\right)+\left(\nabla \cdot u_{h}, \psi\right)=\left(f\left(p_{h}\right), \psi\right), & \forall \psi \in W_{h},
\end{array}
$$

where the approximate saturation $s_{h}: J \rightarrow M_{h}+s_{D}$ is given by

$$
\begin{gathered}
\left(\phi \frac{\partial s_{h}}{\partial t}, v\right)+\left(D\left(s_{h}\right) \nabla s_{h}-q_{w}\left(s_{h}\right) u_{h}-b\left(s_{h}, p_{h}\right), \nabla v\right) \\
=\left(\tilde{f}_{w}-s_{h} \frac{\partial \phi}{\partial t}, v\right)-\left\langle d_{w}, v\right\rangle_{\Gamma_{2}}, \quad \forall v \in M_{h} .
\end{gathered}
$$

The initial conditions satisfy

$$
\begin{aligned}
& p_{h}(\cdot, 0)=0, \\
& s_{h}(\cdot, 0)=0, \\
& u_{h}(\cdot, 0)=0 .
\end{aligned}
$$

We now make a remark about existence and uniqueness of the approximate solution to the nonlinear system (4.5) and (4.6). Introducing bases in $V_{h}, W_{h}$, and $M_{h}$, the problem (4.5) can be written in matrix form as

$$
\begin{aligned}
& A(S) U-B P=G(S, P), \\
& C(S, P) \frac{d P}{d t}+B^{t} U=F(P),
\end{aligned}
$$

with $P(0)$ given, where $A(S)$ and $C(S, P)$ are positive definite by (2.18) and (2.20) and $S$, $U$, and $P$ are the respective degrees of freedom of $s_{h}, u_{h}$, and $p_{h}$. Substituting the relation

$$
U=A(S)^{-1} B P+A(S)^{-1} G(S, P),
$$

into $(4.8 \mathrm{~b})$, we see that

$$
C(S, P) \frac{d P}{d t}+B^{t} A(S)^{-1} B P+B^{t} A(S)^{-1} G(S, P)=F(P),
$$

which, in turn, produces the system

$$
\frac{d P}{d t}=F_{1}(P, S)
$$

Also, using (4.19), it follows from (4.6) that

$$
\frac{d S}{d t}=F_{2}(P, S)
$$

with $S(0)$ given. Now, (4.10) and (4.11) can be treated as a nonlinear system of ordinary differential equations for $(P, S)$, which has a unique solution, at least locally. In fact, since we assumed that the coefficients in (4.5) and (4.6) are smooth, the vector valued function $\left(F_{1}, F_{2}\right)$ is globally Lipschitz continuous, and the solution $(P(t), S(t))$ exists for all positive time $t$. 


\section{Error analyses for the semi-discrete scheme}

In this section we give a convergence analysis for the finite element procedure (4.5) and (4.6). As usual, it is convenient to use an elliptic projection of the solution of (2.11) into the finite element space $M_{h}$. Let $\tilde{s}=\tilde{s}_{h}: J \rightarrow M_{h}+s_{D}$ be defined by

$$
(D(s) \nabla(s-\tilde{s}), \nabla v)+(s-\tilde{s}, v)=0, \quad \forall v \in M_{h}, t \in J .
$$

Set

$$
\zeta=s-\tilde{s}, \quad \xi=\tilde{s}-s_{h}
$$

Then it follows from standard results of the finite element method [13], [25], [31] that

$$
\begin{aligned}
& \|\zeta\|+h\|\zeta\|_{1} \leq C\left(\sum_{K} h_{K}^{2(k+1)}\|s\|_{k+1, K}^{2}\right)^{1 / 2}, \\
& \|\zeta\|_{0, \infty} \leq C h^{k+1}\left(\log h^{-1}\right)^{\gamma}\|s\|_{k+1, \infty}
\end{aligned}
$$

where $\gamma=1$ for $k=1$ and $\gamma=0$ for $k>1$. The same result applies to the timedifferentiated forms of (5.1) [33]:

$$
\left\|\frac{\partial \zeta}{\partial t}\right\|+h\left\|\frac{\partial \zeta}{\partial t}\right\|_{1} \leq C\left(\sum_{K} h_{K}^{2(k+1)}\left(\|s\|_{k+1, K}^{2}+\left\|\frac{\partial s}{\partial t}\right\|_{k+1, K}^{2}\right)\right)^{1 / 2}
$$

As for the analysis of the mixed finite element method, we use the the following two projections instead of the elliptic projections introduced in [12] and [15]. So the present analysis is different from and simpler than those in [12] and [15]. Each of our mixed finite element spaces [2]-[4], [10], [24], [28] has the property that there are projection operators $\Pi_{h}: H^{1}(\Omega) \rightarrow V_{h}$ and $P_{h}=L^{2}$-projection: $L^{2}(\Omega) \rightarrow W_{h}$ such that

$$
\begin{array}{ll}
\left\|v-\Pi_{h} v\right\| \leq C\left(\sum_{K} h_{p, K}^{2 r}\|v\|_{r, K}^{2}\right)^{1 / 2}, & 0 \leq r \leq k^{*}+1, \\
\left\|\nabla \cdot\left(v-\Pi_{h} v\right)\right\| \leq C\left(\sum_{K} h_{p, K}^{2 r}\|\nabla \cdot v\|_{r, K}^{2}\right)^{1 / 2}, & 0 \leq r \leq k^{* *}, \\
\left\|w-P_{h} w\right\| \leq C\left(\sum_{K} h_{p, K}^{2 r}\|w\|_{r, K}^{2}\right)^{1 / 2}, & 0 \leq r \leq k^{* *},
\end{array}
$$

and (see, e.g., [7], [16])

$$
\begin{array}{ll}
\left(\nabla \cdot\left(v-\Pi_{h} v\right), w\right)=0, & \forall w \in W_{h} \\
\left(\nabla \cdot v, w-P_{h} w\right)=0, & \forall v \in V_{h} .
\end{array}
$$

Set $\tilde{p}=P_{h} p, \tilde{u}=\Pi_{h} u$, and

$$
\begin{aligned}
\sigma=u-\tilde{u}, & \beta=\tilde{u}-u_{h} \\
\eta=p-\tilde{p}, & \theta=\tilde{p}-p_{h} .
\end{aligned}
$$


Note that, by (3.3) and (4.7),

$$
\begin{aligned}
& \theta(x, 0)=0 \\
& \xi(x, 0)=0, \\
& \beta(x, 0)=0 .
\end{aligned}
$$

Finally, we prove some bounds of the projections $\tilde{s}$ and $\tilde{p}$. Let $\bar{s}=\bar{s}_{h}$ be the interpolant of $s$ in $M_{h}$. Then we see, by (4.1), (5.3b), and an inverse inequality in $M_{h}$, that

$$
\begin{aligned}
\|\tilde{s}\|_{1, \infty} & \leq\|s-\tilde{s}\|_{1, \infty}+\|s\|_{1, \infty} \\
& \leq\|\bar{s}-\tilde{s}\|_{1, \infty}+\|s-\bar{s}\|_{1, \infty}+\|s\|_{1, \infty} \\
& \leq C h^{-1}\|\bar{s}-\tilde{s}\|_{0, \infty}+\|s-\bar{s}\|_{1, \infty}+\|s\|_{1, \infty} \\
& \leq C h^{-1}\left(\|\tilde{s}-s\|_{0, \infty}+\|s-\bar{s}\|_{0, \infty}\right)+\|s-\bar{s}\|_{1, \infty}+\|s\|_{1, \infty} \\
& \leq C h^{k}\left(\log h^{-1}\right)^{\gamma}\|s\|_{k+1, \infty}+\|s\|_{1, \infty}
\end{aligned}
$$

where $\gamma$ is given as in (5.3b). This implies that $\|\tilde{s}\|_{1, \infty}$ is bounded for sufficiently smooth solutions since $k \geq 1$. The same argument applies to $\|\partial \tilde{s} / \partial t\|_{1, \infty}$. Next, note that, by the approximation property of the projection $P_{h}[22]$,

$$
\left\|\tilde{p}_{t}\right\|_{0, \infty} \leq C\left\|p_{t}\right\|_{0, \infty}
$$

These bounds on $\tilde{p}_{t}, \nabla \tilde{s}$, and $\nabla(\partial \tilde{s} / \partial t)$ are used below.

We are now ready to prove some results. Below $\varepsilon$ is a generic positive constant as small as we please.

5.1. Analysis of the mixed method. We first analyze the mixed method (4.5). The following error equation is obtained by subtracting (4.5) from (3.1) and applying (5.8) and $(5.9)$ :

$$
\begin{aligned}
\left(\alpha\left(s_{h}\right) \beta, v\right)-(\nabla \cdot v, \theta)= & \left(\left(\alpha\left(s_{h}\right)-\alpha(s)\right) u, v\right)-\left(\alpha\left(s_{h}\right) \sigma, v\right) \\
& +\left(G(s, p)-G\left(s_{h}, p_{h}\right), v\right), \quad \forall v \in V_{h}, \\
\left(c\left(s_{h}, p_{h}\right) \frac{\partial \theta}{\partial t}, \psi\right)+(\nabla \cdot \beta, \psi)= & \left(f(p)-f\left(p_{h}\right), \psi\right) \\
& +\left(\left(c\left(s_{h}, p_{h}\right)-c(s, p)\right) \frac{\partial p}{\partial t}, \psi\right) \\
& -\left(c\left(s_{h}, p_{h}\right) \frac{\partial \eta}{\partial t}, \psi\right), \quad \forall \psi \in W_{h} .
\end{aligned}
$$

Lemma 5.1. Let $(u, p)$ and $\left(u_{h}, p_{h}\right)$ satisfy (3.1) and (4.5), respectively. Then

$$
\left\|\frac{\partial \theta}{\partial t}(0)\right\| \leq C\left\|\frac{\partial \eta}{\partial t}(0)\right\| .
$$

Proof. (5.15) follows from setting $\psi=\partial \theta / \partial t$ at $t=0$ in (5.14) and using (3.3) and (4.7). 
Lemma 5.2. It holds that

$$
\begin{gathered}
\left\|\frac{\partial \theta}{\partial t}(t)\right\|^{2}+\int_{0}^{t}\left\|\frac{\partial \beta}{\partial t}(\tau)\right\|^{2} d \tau \\
\leq C_{1}\left(\left\|\frac{\partial \theta}{\partial t}(0)\right\|^{2}+\int_{0}^{t}\left\{\left\|\frac{\partial \sigma}{\partial t}\right\|^{2}+\|\eta\|^{2}+\left\|\frac{\partial \eta}{\partial t}\right\|^{2}+\|\zeta\|^{2}+\left\|\frac{\partial \zeta}{\partial t}\right\|^{2}+\left\|\frac{\partial^{2} \eta}{\partial t^{2}}\right\|^{2}\right\} d \tau\right. \\
+\int_{0}^{t}\left\{\|\xi\|^{2}+\|\theta\|^{2}+\left(1+\left\|\frac{\partial \xi}{\partial t}\right\|_{0, \infty}^{2}\right)\left(\|\beta\|^{2}+\|\sigma\|^{2}\right)\right. \\
\left.\left.\quad+\left\|\frac{\partial \xi}{\partial t}\right\|^{2}+\left(1+\left\|\frac{\partial \xi}{\partial t}\right\|_{0, \infty}^{2}+\left\|\frac{\partial \theta}{\partial t}\right\|_{0, \infty}^{2}\right)\left\|\frac{\partial \theta}{\partial t}\right\|^{2}\right\} d \tau\right), \quad t \in J,
\end{gathered}
$$

where

$$
C_{1}=C_{1}\left(\left\|\frac{\partial \tilde{s}}{\partial t}\right\|_{L^{\infty}(J \times \Omega)},\left\|\frac{\partial p}{\partial t}\right\|_{L^{\infty}(J \times \Omega)},\left\|\frac{\partial^{2} p}{\partial t^{2}}\right\|_{L^{\infty}(J \times \Omega)},\|u\|_{L^{\infty}(J \times \Omega)},\left\|\frac{\partial u}{\partial t}\right\|_{L^{\infty}(J \times \Omega)}\right) .
$$

Proof. Differentiate equations (5.13) and (5.14) with respect to $t$ to have

$$
\begin{aligned}
\left(\alpha\left(s_{h}\right) \frac{\partial \beta}{\partial t}, v\right)-\left(\nabla \cdot v, \frac{\partial \theta}{\partial t}\right)= & \left(\left(\alpha\left(s_{h}\right)-\alpha(s)\right) \frac{\partial u}{\partial t}, v\right) \\
& -\left(\alpha\left(s_{h}\right) \frac{\partial \sigma}{\partial t}, v\right)-\left(\frac{\partial \alpha}{\partial t}\left(s_{h}\right) \sigma, v\right) \\
& +\left(\frac{\partial}{\partial t}\left(\alpha\left(s_{h}\right)-\alpha(s)\right) u, v\right) \\
& +\left(\frac{\partial}{\partial t}\left(G(s, p)-G\left(s_{h}, p_{h}\right)\right), v\right) \\
& -\left(\frac{\partial \alpha}{\partial t}\left(s_{h}\right) \beta, v\right), \quad \forall v \in V_{h},
\end{aligned}
$$

and

$$
\begin{aligned}
\left(c\left(s_{h}, p_{h}\right) \frac{\partial^{2} \theta}{\partial t^{2}}, \psi\right)+\left(\nabla \cdot \frac{\partial \beta}{\partial t}, \psi\right)= & \left(\frac{\partial}{\partial t}\left(f(p)-f\left(p_{h}\right)\right), \psi\right) \\
& -\left(\frac{\partial c}{\partial t}\left(s_{h}, p_{h}\right) \frac{\partial \theta}{\partial t}, \psi\right) \\
& +\left(\left(c\left(s_{h}, p_{h}\right)-c(s, p)\right) \frac{\partial^{2} p}{\partial t^{2}}, \psi\right) \\
& +\left(\frac{\partial}{\partial t}\left(c\left(s_{h}, p_{h}\right)-c(s, p)\right) \frac{\partial p}{\partial t}, \psi\right) \\
& -\left(c\left(s_{h}, p_{h}\right) \frac{\partial^{2} \eta}{\partial t^{2}}, \psi\right) \\
& -\left(\frac{\partial c}{\partial t}\left(s_{h}, p_{h}\right) \frac{\partial \eta}{\partial t}, \psi\right), \quad \forall \psi \in W_{h} .
\end{aligned}
$$

Set $v=\partial \beta / \partial t$ in (5.17) and $\psi=\partial \theta / \partial t$ in (5.18) to find that

$$
\left(\alpha\left(s_{h}\right) \frac{\partial \beta}{\partial t}, \frac{\partial \beta}{\partial t}\right)+\frac{1}{2} \frac{d}{d t}\left(c\left(s_{h}, p_{h}\right) \frac{\partial \theta}{\partial t}, \frac{\partial \theta}{\partial t}\right)=\sum_{i=1}^{12} T_{i}
$$


where

$$
\begin{aligned}
T_{1} & =-\left(\frac{\partial \alpha}{\partial t}\left(s_{h}\right) \beta, \frac{\partial \beta}{\partial t}\right), & T_{2} & =\left(\frac{\partial G}{\partial t}(s, p)-\frac{\partial G}{\partial t}\left(s_{h}, p_{h}\right), \frac{\partial \beta}{\partial t}\right), \\
T_{3} & =\left(\left(\alpha\left(s_{h}\right)-\alpha(s)\right) \frac{\partial u}{\partial t}, \frac{\partial \beta}{\partial t}\right), & T_{4} & =\left(\left(\frac{\partial \alpha}{\partial t}\left(s_{h}\right)-\frac{\partial \alpha}{\partial t}(s)\right) u, \frac{\partial \beta}{\partial t}\right), \\
T_{5} & =-\left(\frac{\partial \alpha}{\partial t}\left(s_{h}\right) \sigma, \frac{\partial \beta}{\partial t}\right), & T_{6} & =-\left(\alpha\left(s_{h}\right) \frac{\partial \sigma}{\partial t}, \frac{\partial \beta}{\partial t}\right), \\
T_{7} & =\left(\frac{\partial}{\partial t}\left(f(p)-f\left(p_{h}\right)\right), \frac{\partial \theta}{\partial t}\right), & T_{8} & =\left(\frac{\partial}{\partial t}\left(c\left(s_{h}, p_{h}\right)-c(s, p)\right) \frac{\partial p}{\partial t}, \frac{\partial \theta}{\partial t}\right), \\
T_{9} & =-\left(\frac{\partial c}{\partial t}\left(s_{h}, p_{h}\right) \frac{\partial \eta}{\partial t}, \frac{\partial \theta}{\partial t}\right), & T_{10} & =-\frac{1}{2}\left(\frac{\partial c}{\partial t}\left(s_{h}, p_{h}\right) \frac{\partial \theta}{\partial t}, \frac{\partial \theta}{\partial t}\right), \\
T_{11} & =\left(\left(c\left(s_{h}, p_{h}\right)-c(s, p)\right) \frac{\partial^{2} p}{\partial t^{2}}, \frac{\partial \theta}{\partial t}\right), & T_{12} & =-\left(c\left(s_{h}, p_{h}\right) \frac{\partial^{2} \eta}{\partial t^{2}}, \frac{\partial \theta}{\partial t}\right) .
\end{aligned}
$$

It is easily seen that

$$
\left|T_{3}\right|+\left|T_{6}\right| \leq C_{1}\left(\|\xi\|^{2}+\|\zeta\|^{2}+\left\|\frac{\partial \sigma}{\partial t}\right\|^{2}\right)+\varepsilon\left\|\frac{\partial \beta}{\partial t}\right\|^{2}
$$

Next, note that

$$
T_{1}=\frac{1}{2}\left(\alpha^{\prime}\left(s_{h}\right) \frac{\partial \xi}{\partial t} \beta, \frac{\partial \beta}{\partial t}\right)-\frac{1}{2}\left(\alpha^{\prime}\left(s_{h}\right) \frac{\partial \tilde{s}}{\partial t} \beta, \frac{\partial \beta}{\partial t}\right),
$$

so that

$$
\left|T_{1}\right| \leq C_{1}\left(1+\left\|\frac{\partial \xi}{\partial t}\right\|_{0, \infty}^{2}\right)\|\beta\|^{2}+\varepsilon\left\|\frac{\partial \beta}{\partial t}\right\|^{2}
$$

Similarly, we see that

$$
\begin{gathered}
\left|T_{2}\right| \leq C_{1}\left(\|\xi\|^{2}+\|\zeta\|^{2}+\|\eta\|^{2}+\|\theta\|^{2}+\left\|\frac{\partial \xi}{\partial t}\right\|^{2}\right. \\
\left.+\left\|\frac{\partial \zeta}{\partial t}\right\|^{2}+\left\|\frac{\partial \eta}{\partial t}\right\|^{2}+\left\|\frac{\partial \theta}{\partial t}\right\|^{2}\right)+\varepsilon\left\|\frac{\partial \beta}{\partial t}\right\|^{2} \\
\left|T_{4}\right| \leq C_{1}\left(\|\xi\|^{2}+\|\zeta\|^{2}+\left\|\frac{\partial \zeta}{\partial t}\right\|^{2}+\left\|\frac{\partial \xi}{\partial t}\right\|^{2}\right)+\varepsilon\left\|\frac{\partial \beta}{\partial t}\right\|^{2} \\
\left|T_{5}\right| \leq C_{1}\left(1+\left\|\frac{\partial \xi}{\partial t}\right\|_{0, \infty}^{2}\right)\|\sigma\|^{2}+\varepsilon\left\|\frac{\partial \beta}{\partial t}\right\|^{2} \\
\left|T_{7}\right| \leq C_{1}\left(\|\eta\|^{2}+\|\theta\|^{2}+\left\|\frac{\partial \eta}{\partial t}\right\|^{2}+\left\|\frac{\partial \theta}{\partial t}\right\|^{2}\right) \\
\left|T_{8}\right| \leq C_{1}\left(\|\xi\|^{2}+\|\zeta\|^{2}+\|\eta\|^{2}+\|\theta\|^{2}\right. \\
\left.\quad+\left\|\frac{\partial \xi}{\partial t}\right\|^{2}+\left\|\frac{\partial \zeta}{\partial t}\right\|^{2}+\left\|\frac{\partial \eta}{\partial t}\right\|^{2}+\left\|\frac{\partial \theta}{\partial t}\right\|^{2}\right) \\
\left|T_{9}\right| \leq C_{1}\left(\left\|\frac{\partial \eta}{\partial t}\right\|^{2}+\left(1+\left\|\frac{\partial \xi}{\partial t}\right\|_{0, \infty}^{2}+\left\|\frac{\partial \theta}{\partial t}\right\|_{0, \infty}^{2}\right)\left\|\frac{\partial \theta}{\partial t}\right\|^{2}\right) \\
\left|T_{10}\right| \leq C_{1}\left(1+\left\|\frac{\partial \xi}{\partial t}\right\|_{0, \infty}^{2}+\left\|\frac{\partial \theta}{\partial t}\right\|_{0, \infty}^{2}\right)\left\|\frac{\partial \theta}{\partial t}\right\|^{2}
\end{gathered}
$$




$$
\begin{aligned}
& \left|T_{11}\right| \leq C_{1}\left(\|\xi\|^{2}+\|\zeta\|^{2}+\|\eta\|^{2}+\|\theta\|^{2}+\left\|\frac{\partial \theta}{\partial t}\right\|^{2}\right), \\
& \left|T_{12}\right| \leq C_{1}\left(\left\|\frac{\partial^{2} \eta}{\partial t^{2}}\right\|^{2}+\left\|\frac{\partial \theta}{\partial t}\right\|^{2}\right) .
\end{aligned}
$$

Now, integrate (5.19) on $t$, substitute (5.20)-(5.30) into the resulting inequality, and use (2.18) and (2.20) to get the result (5.16).

The error equations (5.13) and (5.14) are usually exploited to derive error estimates in the parabolic mixed finite element method [8], [15], [22]. To handle the difficulty arising from the combination of the Dirichlet boundary condition (1.3) and the nonlinearity of the differential system (2.9)-(2.11), we must use the time-differentiated forms (5.17) and (5.18).

5.2. Analysis of the saturation equation. We now turn to analyzing the finite element method (4.6).

Lemma 5.3. Let $s$ and $s_{h}$ satisfy (3.2) and (4.6), respectively. Then

$$
\begin{aligned}
\int_{0}^{t} \| & \frac{\partial \xi}{\partial t}\left\|^{2} d \tau+\right\| \nabla \xi(t) \|^{2} \\
\leq & C_{2}\left\{\int_{0}^{t}\left(\|\zeta\|^{2}+\|\eta\|^{2}+\|\sigma\|^{2}+\left\|\frac{\partial \sigma}{\partial t}\right\|^{2}+\left\|\frac{\partial \zeta}{\partial t}\right\|^{2}+\left\|\frac{\partial \eta}{\partial t}\right\|^{2}\right) d \tau\right. \\
& +\int_{0}^{t}\left(\|\xi\|_{1}^{2}+\|\theta\|^{2}+\|\beta\|^{2}+\left\|\frac{\partial \theta}{\partial t}\right\|^{2}+\|\nabla \xi\|^{2}\left\|\frac{\partial \xi}{\partial t}\right\|_{0, \infty}^{2}\right) d \tau \\
& \left.+\|\zeta(t)\|^{2}+\|\xi(t)\|^{2}+\|\eta(t)\|^{2}+\|\theta(t)\|^{2}+\|\sigma(t)\|^{2}+\|\beta(t)\|^{2}\right\} \\
& +\varepsilon \int_{0}^{t}\left\|\frac{\partial \beta}{\partial t}\right\|^{2} d \tau, \quad t \in J,
\end{aligned}
$$

where

$$
C_{2}=C_{2}\left(\left\|\frac{\partial s}{\partial t}\right\|_{L^{\infty}(J \times \Omega)},\left\|\nabla \frac{\partial \tilde{s}}{\partial t}\right\|_{L^{\infty}(J \times \Omega)},\|\nabla \tilde{s}\|_{L^{\infty}(J \times \Omega)},\|u\|_{L^{\infty}(J \times \Omega)}\right) .
$$

Proof. Subtraction of (4.6) from (3.2) and use of (5.1) leads to the error equation

$$
\begin{aligned}
\left(\phi \frac{\partial \xi}{\partial t}, v\right)+\left(D\left(s_{h}\right) \nabla \xi, \nabla v\right)= & -\left(\phi \frac{\partial \zeta}{\partial t}, v\right)+(\zeta, v) \\
& -\left(q_{w}\left(s_{h}\right)\left(u-u_{h}\right), \nabla v\right)+\left(\left(q_{w}(s)-q_{w}\left(s_{h}\right)\right) u, \nabla v\right) \\
& +\left(b(s, p)-b\left(s_{h}, p_{h}\right), \nabla v\right)-\left(\frac{\partial \phi}{\partial t}\left(s-s_{h}\right), v\right) \\
& -\left(\left(D(s)-D\left(s_{h}\right)\right) \nabla \tilde{s}, \nabla v\right), \quad \forall v \in M_{h} .
\end{aligned}
$$

Take the test function $v=\partial \xi / \partial t$ in (5.32) and write the resulting equation as follows:

$$
\left(\phi \frac{\partial \xi}{\partial t}, \frac{\partial \xi}{\partial t}\right)+\frac{1}{2} \frac{d}{d t}\left(D\left(s_{h}\right) \nabla \xi, \nabla \xi\right)=\sum_{i=1}^{6} B_{i}+\frac{1}{2}\left(\frac{\partial D}{\partial t}\left(s_{h}\right) \nabla \xi, \nabla \xi\right)
$$


where

$$
\begin{array}{ll}
B_{1}=\left(\zeta-\phi \frac{\partial \zeta}{\partial t}, \frac{\partial \xi}{\partial t}\right), & B_{2}=-\left(q_{w}\left(s_{h}\right)\left(u-u_{h}\right), \nabla \frac{\partial \xi}{\partial t}\right) \\
B_{3}=\left(\left(q_{w}(s)-q_{w}\left(s_{h}\right)\right) u, \nabla \frac{\partial \xi}{\partial t}\right), & B_{4}=\left(b(s, p)-b\left(s_{h}, p_{h}\right), \nabla \frac{\partial \xi}{\partial t}\right) \\
B_{5}=-\left(\frac{\partial \phi}{\partial t}\left(s-s_{h}\right), \frac{\partial \xi}{\partial t}\right), & B_{6}=-\left(\left(D(s)-D\left(s_{h}\right)\right) \nabla \tilde{s}, \nabla \frac{\partial \xi}{\partial t}\right) .
\end{array}
$$

It can be easily seen that

$$
\left|B_{1}\right|+\left|B_{5}\right| \leq C_{2}\left(\|\xi\|^{2}+\|\zeta\|^{2}+\left\|\frac{\partial \zeta}{\partial t}\right\|^{2}\right)+\varepsilon\left\|\frac{\partial \xi}{\partial t}\right\|^{2} .
$$

Next, to avoid an apparent loss of a factor $h$, we use integration by parts in time to see that

$$
\begin{aligned}
B_{2}= & -\frac{d}{d t}\left(q_{w}\left(s_{h}\right)\left(u-u_{h}\right), \nabla \xi\right)+\left(\frac{\partial q_{w}}{\partial t}\left(s_{h}\right)\left(u-u_{h}\right), \nabla \xi\right) \\
& +\left(q_{w}\left(s_{h}\right) \frac{\partial}{\partial t}\left(u-u_{h}\right), \nabla \xi\right),
\end{aligned}
$$

so that

$$
\begin{aligned}
\left|\int_{0}^{t} B_{2} d \tau\right| \leq & C_{2}\left\{\|\sigma(t)\|^{2}+\|\beta(t)\|^{2}\right. \\
& \left.+\int_{0}^{t}\left(\left(1+\left\|\frac{\partial \xi}{\partial t}\right\|_{0, \infty}^{2}\right)\|\nabla \xi\|^{2}+\|\sigma\|^{2}+\|\beta\|^{2}+\left\|\frac{\partial \sigma}{\partial t}\right\|^{2}\right) d \tau\right\} \\
+ & \varepsilon\left(\|\nabla \xi(t)\|^{2}+\int_{0}^{t}\left\|\frac{\partial \beta}{\partial t}\right\|^{2} d \tau\right) .
\end{aligned}
$$

Analogously, we have

$$
\begin{aligned}
\left|\int_{0}^{t}\left(B_{3}+B_{6}\right) d \tau\right| \leq & C_{2}\left\{\|\xi(t)\|^{2}+\|\zeta(t)\|^{2}\right. \\
& \left.+\int_{0}^{t}\left(\|\xi\|_{1}^{2}+\|\zeta\|^{2}+\left\|\frac{\partial \zeta}{\partial t}\right\|^{2}\right) d \tau\right\} \\
+ & \varepsilon\left(\|\nabla \xi(t)\|^{2}+\int_{0}^{t}\left\|\frac{\partial \xi}{\partial t}\right\|^{2} d \tau\right),
\end{aligned}
$$

and

$$
\begin{aligned}
\left|\int_{0}^{t} B_{4} d \tau\right| \leq & C_{2}\left\{\|\xi(t)\|^{2}+\|\zeta(t)\|^{2}+\|\theta(t)\|^{2}+\|\eta(t)\|^{2}\right. \\
& \left.+\int_{0}^{t}\left(\|\xi\|_{1}^{2}+\|\zeta\|^{2}+\|\theta\|^{2}+\|\eta\|^{2}+\left\|\frac{\partial \zeta}{\partial t}\right\|^{2}+\left\|\frac{\partial \theta}{\partial t}\right\|^{2}+\left\|\frac{\partial \eta}{\partial t}\right\|^{2}\right) d \tau\right\} \\
+ & \varepsilon\left(\|\nabla \xi(t)\|^{2}+\int_{0}^{t}\left\|\frac{\partial \xi}{\partial t}\right\|^{2} d \tau\right)
\end{aligned}
$$


Finally, since

$$
\left(\frac{\partial D}{\partial t}\left(s_{h}\right) \nabla \xi, \nabla \xi\right)=-\left(\frac{\partial \xi}{\partial t} D^{\prime}\left(s_{h}\right) \nabla \xi, \nabla \xi\right)+\left(\frac{\partial \tilde{s}}{\partial t} D^{\prime}\left(s_{h}\right) \nabla \xi, \nabla \xi\right),
$$

we see that

$$
\left|\left(\frac{\partial D}{\partial t}\left(s_{h}\right) \nabla \xi, \nabla \xi\right)\right| \leq C_{2}\left(1+\left\|\frac{\partial \xi}{\partial t}\right\|_{0, \infty}^{2}\right)\|\nabla \xi\|^{2} .
$$

Now, integrate (5.33) on $t$, substitute (5.33)-(5.38) into the resulting inequality, and use (2.19), (2.21), and (5.12b) to get the result (5.31).

5.3. $L^{2}$-error estimates. We now state the main result in this section. Define

$$
\begin{aligned}
\mathcal{E}(t)= & \sum_{K \in T_{h_{p}}} h_{p, K}^{k^{* *}}\left(\|p\|_{L^{\infty}\left(0, t ; H^{\left.k^{* *}(K)\right)}\right.}+\left\|\frac{\partial p}{\partial t}\right\|_{L^{\infty}\left(0, t ; H^{\left.k^{* *}(K)\right)}\right.}+\left\|\frac{\partial^{2} p}{\partial t^{2}}\right\|_{L^{2}\left(0, t ; H^{k^{* *}}(K)\right)}\right) \\
& +\sum_{K \in T_{h_{p}}} h_{p, K}^{k^{*}+1}\left(\|u\|_{L^{\infty}\left(0, t ; H^{k^{*}+1}(K)\right)}+\left\|\frac{\partial u}{\partial t}\right\|_{L^{2}\left(0, t ; H^{k^{*}+1}(K)\right)}\right) \\
& +\sum_{K \in T_{h}} h_{K}^{k+1}\left(\|s\|_{L^{\infty}\left(0, t ; H^{k+1}(K)\right)}+\left\|\frac{\partial s}{\partial t}\right\|_{L^{2}\left(0, t ; H^{k+1}(K)\right)}\right), \quad t \in J .
\end{aligned}
$$

Theorem 5.4. Let $(u, p, s)$ and $\left(u_{h}, p_{h}, s_{h}\right)$ satisfy (3.1), (3.2) and (4.5), (4.6), respectively. Then, if the parameters $h_{p}$ and $h$ satisfy

$$
\left(h^{-d / 2}+h_{p}^{-d / 2}\right)\left(h_{p}^{k^{*}+1}+h_{p}^{k^{* *}}+h^{k+1}\right) \rightarrow 0 \text { as } h \rightarrow 0,
$$

we have

$$
\begin{aligned}
\| u & -u_{h}\left\|_{L^{\infty}\left(J ; L^{2}(\Omega)\right)}+\right\| p-p_{h} \|_{L^{\infty}\left(J ; L^{2}(\Omega)\right)} \\
& +\left\|\frac{\partial p}{\partial t}-\frac{\partial p_{h}}{\partial t}\right\|_{L^{\infty}\left(J ; L^{2}(\Omega)\right)}+\left\|s-s_{h}\right\|_{L^{\infty}\left(J ; L^{2}(\Omega)\right)} \\
& +h\left\|s-s_{h}\right\|_{L^{\infty}\left(J ; H^{1}(\Omega)\right)}+\left\|\frac{\partial s}{\partial t}-\frac{\partial s_{h}}{\partial t}\right\|_{L^{2}\left(J ; L^{2}(\Omega)\right)} \leq \mathcal{C E}(T),
\end{aligned}
$$

where $\mathcal{C}=\mathcal{C}\left(C_{1}, C_{2}, T\right)$.

Proof. Take a $\left(C_{1}+1\right)$-multiple of (5.31), add the resulting inequality and (5.16), and use (5.3)-(5.7) and (5.15) to obtain

$$
\begin{aligned}
&\left\|\frac{\partial \theta}{\partial t}(t)\right\|^{2}+\|\nabla \xi(t)\|^{2}+\int_{0}^{t}\left(\left\|\frac{\partial \beta}{\partial t}\right\|^{2}+\left\|\frac{\partial \xi}{\partial t}\right\|^{2}\right) d \tau \\
& \leq C_{3}\left\{\mathcal{E}^{2}(t)+\|\xi(t)\|^{2}+\|\theta(t)\|^{2}+\|\beta(t)\|^{2}\right. \\
&+\int_{0}^{t}\left(\|\xi\|_{1}^{2}+\|\beta\|^{2}+\|\theta\|^{2}+\left\|\frac{\partial \theta}{\partial t}\right\|^{2}\right. \\
&\left.\left.+\left(\|\nabla \xi\|^{2}+\|\beta\|^{2}+\|\sigma\|^{2}+\left\|\frac{\partial \theta}{\partial t}\right\|^{2}\right)\left(\left\|\frac{\partial \xi}{\partial t}\right\|_{0, \infty}^{2}+\left\|\frac{\partial \theta}{\partial t}\right\|_{0, \infty}^{2}\right)\right) d \tau\right\}
\end{aligned}
$$


where $C_{3}=C_{3}\left(C_{1}, C_{2}\right)$. In deriving (5.40), we required that the $\varepsilon$ appearing in (5.31) be sufficiently small that $\left(C_{1}+1\right) \varepsilon \leq 1 / 2$; this increases $C_{2}$, but not $C_{1}$. Observe that

$$
\|\xi(t)\|^{2}=\int_{0}^{t} \frac{d}{d t}\|\xi(\tau)\|^{2} d \tau \leq C \int_{0}^{t}\|\xi\|^{2} d \tau+\varepsilon \int_{0}^{t}\left\|\frac{\partial \xi}{\partial t}\right\|^{2} d \tau
$$

the same result holds for $\beta$ and $\theta$. Apply (5.40) and (5.41) to see that

$$
\begin{aligned}
&\left\|\frac{\partial \theta}{\partial t}(t)\right\|^{2}+\|\xi(t)\|_{1}^{2}+\|\beta(t)\|^{2}+\|\theta(t)\|^{2}+\int_{0}^{t}\left(\left\|\frac{\partial \beta}{\partial t}\right\|^{2}+\left\|\frac{\partial \xi}{\partial t}\right\|^{2}\right) d \tau \\
& \leq C_{3}\left\{\mathcal{E}^{2}(t)+\int_{0}^{t}\left(\|\xi\|_{1}^{2}+\|\beta\|^{2}+\|\theta\|^{2}+\left\|\frac{\partial \theta}{\partial t}\right\|^{2}\right.\right. \\
&\left.\left.+\left(\|\nabla \xi\|^{2}+\|\beta\|^{2}+\|\sigma\|^{2}+\left\|\frac{\partial \theta}{\partial t}\right\|^{2}\right)\left(\left\|\frac{\partial \xi}{\partial t}\right\|_{0, \infty}^{2}+\left\|\frac{\partial \theta}{\partial t}\right\|_{0, \infty}^{2}\right)\right) d \tau\right\}
\end{aligned}
$$

We now make the induction hypothesis that

$$
\begin{aligned}
\left(\|\nabla \xi\|_{L^{\infty}\left(J ; L^{2}(\Omega)\right)}+\right. & \left.\|\beta\|_{L^{\infty}\left(J ; L^{2}(\Omega)\right)}+\left\|\frac{\partial \theta}{\partial t}\right\|_{L^{\infty}\left(J ; L^{2}(\Omega)\right)}+\mathcal{E}(T)\right) \\
& \times\left(\left\|\frac{\partial \xi}{\partial t}\right\|_{L^{2}\left(J ; L^{\infty}(\Omega)\right)}+\left\|\frac{\partial \theta}{\partial t}\right\|_{L^{\infty}\left(J ; L^{\infty}(\Omega)\right)}\right) \leq C_{4} \mathcal{E}(T) .
\end{aligned}
$$

Then it follows from the Gronwall lemma that

$$
\begin{aligned}
& \|\theta\|_{L^{\infty}\left(J ; L^{2}(\Omega)\right)}+\|\beta\|_{L^{\infty}\left(J ; L^{2}(\Omega)\right)}+\|\xi\|_{L^{\infty}\left(J ; H^{1}(\Omega)\right)} \\
& \quad+\left\|\frac{\partial \theta}{\partial t}\right\|_{L^{\infty}\left(J ; L^{2}(\Omega)\right)}+\left\|\frac{\partial \xi}{\partial t}\right\|_{L^{2}\left(J ; L^{2}(\Omega)\right)} \leq C_{5} \mathcal{E}(T)
\end{aligned}
$$

where $C_{5}=C_{5}\left(C_{1}, C_{2}, C_{4}\right)$. Now the theorem follows from (5.3)-(5.7) and (5.44). It thus remains to prove the induction hypothesis (5.43). Obviously, it holds for $t=0$ from the choices of the approximate initial data. Set

$$
\begin{aligned}
F(t)= & \left(\|\nabla \xi\|_{L^{\infty}\left(0, t ; L^{2}(\Omega)\right)}+\|\beta\|_{L^{\infty}\left(0, t ; L^{2}(\Omega)\right)}+\left\|\frac{\partial \theta}{\partial t}\right\|_{L^{\infty}\left(0, t ; L^{2}(\Omega)\right)}+\mathcal{E}(T)\right) \\
& \times\left(\left\|\frac{\partial \xi}{\partial t}\right\|_{L^{2}\left(0, t ; L^{\infty}(\Omega)\right)}+\left\|\frac{\partial \theta}{\partial t}\right\|_{L^{\infty}\left(0, t ; L^{\infty}(\Omega)\right)}\right) .
\end{aligned}
$$

Since $F(t)$ is continuous in $t$, there is a $t^{*}$ such that

$$
\begin{array}{ll}
F(t)<C_{4} \mathcal{E}(T), & 0<t<t^{*}, \\
F(t)=C_{4} \mathcal{E}(T), & t=t^{*} .
\end{array}
$$

We prove that $t^{*}=T$. Exploiting (5.44) and the inverse inequalities in $M_{h}$ and $W_{h}$

$$
\begin{gathered}
\left\|\frac{\partial \xi}{\partial t}\right\|_{0, \infty} \leq C_{6} h^{-d / 2}\left\|\frac{\partial \xi}{\partial t}\right\|, \\
\left\|\frac{\partial \theta}{\partial t}\right\|_{0, \infty} \leq C_{6} h_{p}^{-d / 2}\left\|\frac{\partial \theta}{\partial t}\right\|,
\end{gathered}
$$


we see that

$$
F(t) \leq 2 C_{5}^{2} C_{6}\left(h^{-d / 2}+h_{p}^{-d / 2}\right) \mathcal{E}^{2}(T), \quad 0 \leq t \leq t^{*} .
$$

By the relation (5.39), $h$ can be selected to be sufficiently small so that

$$
F\left(t^{*}\right) \leq \frac{C_{4}}{2} \mathcal{E}(T)
$$

Therefore, $t^{*}=T$; i.e., (5.43) holds.

We remark that, if $h$ and $h_{p}$ are of the same order as they tend to zero, then

$$
\left(h^{-d / 2}+h_{p}^{-d / 2}\right)\left(h_{p}^{k^{*}+1}+h_{p}^{k^{* *}}+h^{k+1}\right) \leq C h^{-d / 2}\left(h^{k^{* *}}+h^{k+1}\right),
$$

since $k^{* *} \leq k^{*}+1$. Since $k \geq 1$,

$$
h^{-d / 2} h^{k+1} \rightarrow 0 \text { as } h \rightarrow 0, d=2,3 .
$$

Also, if $k^{* *} \geq 2$, we see that

$$
h^{-d / 2} h^{k^{* *}} \rightarrow 0 \text { as } h \rightarrow 0, d=2,3 .
$$

Thus, for (5.39) to be satisfied, we assume that $k^{* *} \geq 2$. This excludes the mixed finite element spaces of lowest order, i.e., $k^{* *}=1$. The lowest order case has to be treated using different techniques. If the nonlinear coefficients $\alpha(s)$ and $c(s, p)$ in (4.5) are projected into the finite element space $W_{h}$, the technique developed in [9] can be used to handle the lowest order case. We shall not pursue this here.

5.4. $L^{\infty}$-error estimates. The main objective of this paper is to establish the $L^{2}$-error estimates given in Theorem 5.4. For completeness, we end this section with a statement of $L^{\infty}$-estimates for the errors $s-s_{h}$ and $p-p_{h}$ in the two-dimensional case.

Theorem 5.5. Assume that $(p, s)$ and $\left(p_{h}, s_{h}\right)$ satisfy (3.1), (3.2) and (4.5), (4.6), respectively, and the parameters $h_{p}$ and $h$ satisfy (5.39). Then

$$
\begin{aligned}
& \left\|p-p_{h}\right\|_{L^{\infty}\left(J ; L^{\infty}(\Omega)\right)} \leq C \log h_{p}^{-1}\left(\mathcal{E}(T)+h_{p}^{k^{* *}}\|p\|_{L^{\infty}\left(J ; H^{k^{* *}+1}(\Omega)\right)}\right), \\
& \left\|s-s_{h}\right\|_{L^{\infty}\left(J ; L^{\infty}(\Omega)\right)} \leq C\left(\log h^{-1}\right)^{\gamma}\left(\mathcal{E}(T)+h^{k+1}\|s\|_{L^{\infty}\left(J ; W^{k+1, \infty}(\Omega)\right)}\right),
\end{aligned}
$$

where $C=C\left(C_{1}, C_{2}, T\right), \gamma=1$ for $k=1$, and $\gamma=1 / 2$ for $k>1$.

Proof. First, it follows from the approximation property of the projection $P_{h}[22]$ that

$$
\|p-\tilde{p}\|_{0, \infty} \leq C h_{p}^{k^{* *}}\left(\log h_{p}^{-1}\right)^{1 / 2}\|p\|_{k^{* *}+1}
$$

Also, from [22, Lemma 1.2] and (5.13), we see that

$$
\|\theta\|_{0, \infty} \leq C \log h_{p}^{-1}\left\|\alpha\left(s_{h}\right) \beta+\left(\alpha(s)-\alpha\left(s_{h}\right)\right) u+\alpha\left(s_{h}\right) \sigma+\left(G\left(s_{h}, p_{h}\right)-G(s, p)\right)\right\|,
$$

so that, by Theorem 5.4,

$$
\|\theta\|_{L^{\infty}\left(J ; L^{\infty}(\Omega)\right)} \leq C \log h_{p}^{-1} \mathcal{E}(T) .
$$

This, together with (5.49), implies (5.47). Finally, apply the embedding inequality [30]

$$
\|\xi\|_{0, \infty} \leq C\left(\log h^{-1}\right)^{1 / 2}\|\xi\|_{1},
$$

(5.3b), and (5.44) to obtain (5.48). 


\section{A degenerate problem}

In this section we consider a degenerate case where the diffusion coefficient $D(s)$ can be zero. Since the pressure equation is the same as before, we here focus on the saturation equation. For simplicity we neglect gravity. Then the saturation equation (2.11) can be written as

$$
\phi \frac{\partial s}{\partial t}-\nabla \cdot\left(D(s) \nabla s-q_{w}(s) u\right)=\tilde{f}_{w}-s \frac{\partial \phi}{\partial t}, \quad(x, t) \in \Omega \times J .
$$

For technical reasons we only consider the Neumann boundary condition (2.15):

$$
\left(D(s) \nabla s-q_{w}(s) u\right) \cdot \nu=-d_{w}(x, t), \quad(x, t) \in \partial \Omega \times J,
$$

and the initial condition is given by

$$
s(x, 0)=s^{0}(x), \quad x \in \Omega,
$$

where $0 \leq s^{0}(x) \leq 1, x \in \Omega$. We impose the following conditions on the degeneracy of $D(s)$ :

$$
D(s) \geq \begin{cases}\beta_{1}|s|^{\mu_{1}}, & 0 \leq s \leq \alpha_{1}, \\ \beta_{2}, & \alpha_{1} \leq s \leq \alpha_{2} \\ \beta_{3}|1-s|^{\mu_{2}}, & \alpha_{2} \leq s \leq 1\end{cases}
$$

where the $\beta_{i}$ are positive constants and $\alpha_{j}$ and $\mu_{j}(j=1,2)$ satisfy the conditions:

$$
0<\alpha_{1}<1 / 2<\alpha_{2}<1,0<\mu_{j} \leq 2
$$

Difficulties arise when trying to derive error estimates for the approximate solution of (6.1) and (6.2) with $D(s)$ satisfying the condition (6.3). To get around this problem, we consider the perturbed diffusion coefficient $D_{\kappa}(s)$ defined by [19], [29], [32]

$$
D_{\kappa}(s)=\max \left\{D(s), \kappa^{\mu}\right\},
$$

where $\kappa>0$ and $\mu=\max \left\{\mu_{1}, \mu_{2}\right\}$. Since the coefficient $D_{\kappa}(s)$ is bounded away from zero, the previous error analysis applies to the perturbed problem:

$$
\begin{array}{ll}
\phi \frac{\partial s_{\kappa}}{\partial t}-\nabla \cdot\left(D_{\kappa}\left(s_{\kappa}\right) \nabla s_{\kappa}-q_{w}\left(s_{\kappa}\right) u\right)=\tilde{f}_{w}-s_{\kappa} \frac{\partial \phi}{\partial t}, & (x, t) \in \Omega \times J, \\
\left(D_{\kappa}\left(s_{\kappa}\right) \nabla s_{\kappa}-q_{w}\left(s_{\kappa}\right) u\right) \cdot \nu=-d_{w}(x, t), & (x, t) \in \partial \Omega \times J, \\
s_{\kappa}(x, 0)=s^{0}(x), & x \in \Omega .
\end{array}
$$

We now state a result on the convergence of $s_{\kappa}$ to $s$ as $\kappa$ tends to zero. Its proof is given in [19] for the case where $d_{w} \equiv 0$ and the right-hand side of (6.1) is zero, and can be easily extended to the present case. 
Theorem 6.1. Assume that $D(s)$ satisfies (6.3) and there is a constant $C^{*}>0$ such that

$$
C^{*}\left|q_{w}\left(s_{1}\right)-q_{w}\left(s_{2}\right)\right|^{2} \leq\left(\mathcal{D}\left(s_{1}\right)-\mathcal{D}\left(s_{2}\right)\right)\left(s_{1}-s_{2}\right), \quad 0 \leq s_{1}, s_{2} \leq 1,
$$

where

$$
\mathcal{D}(s)=\int_{0}^{s} D(\xi) d \xi .
$$

Then there is $C$ independent of $\kappa, s$, and $\mu$ such that

$$
\left\|s-s_{\kappa}\right\|_{L^{2+\mu}\left(J ; L^{2+\mu}(\Omega)\right)} \leq C \kappa
$$

As shown in [19], the requirement (6.5) is reasonable. We now consider the continuoustime finite element method for (6.4). Let $M_{h}$ be the standard $C^{0}$ piecewise linear polynomial space associated with $T_{h}$; due to the roughness of the solution to (6.1) and (6.2), no improvements in the asymptotic convergence rates result from taking higher order finite element spaces. Also, we extend the domain of $D_{\kappa}$ and $q_{w}$ as follows:

$$
D_{\kappa}(\xi)= \begin{cases}D_{\kappa}(1) & \text { if } \xi \geq 1 \\ D_{\kappa}(-\xi) & \text { if } \xi \leq 0\end{cases}
$$

and

$$
q_{w}(\xi)=0, \quad \forall \xi \in(-\infty, 0) \cup(1, \infty) .
$$

The finite element solution $s_{h}: J \rightarrow M_{h}$ to (6.4) is given by

$$
\begin{aligned}
& \left(\phi \frac{\partial s_{h}}{\partial t}, v\right)+\left(D_{\kappa}\left(s_{h}\right) \nabla s_{h}-q_{w}\left(s_{h}\right) u, \nabla v\right) \\
& \quad=\left(\tilde{f}_{w}-s_{h} \frac{\partial \phi}{\partial t}, v\right)-\left\langle d_{w}, v\right\rangle_{\partial \Omega}, \quad \forall v \in M_{h}, \\
& s_{h}(\cdot, 0)=\mathcal{P}_{h} s^{0}
\end{aligned}
$$

where $\mathcal{P}_{h}$ is the $L^{2}$-projection onto $M_{h}$. The following theorem states the convergence of $s_{h}$ to $s$. For (6.8) below to be satisfied, we need that the perturbation parameter $\kappa$ satisfies the relation $\kappa=O\left(h^{\lambda_{1}}\right)$, where $\lambda_{1}$ is given below.

Theorem 6.2. Let $s$ and $s_{h}$ satisfy (6.1), (6.2) and (6.7), respectively, and let the hypotheses of Theorem 6.1 be satisfied. Then there is $C$ independent of $\kappa, s$, and $\mu$ such that

$$
\left\|s-s_{h}\right\|_{L^{2+\mu}\left(J ; L^{2+\mu}(\Omega)\right)} \leq C h^{\lambda_{1}}\left(\log h^{-1}\right)^{\lambda_{2}}
$$

where $\lambda_{1}=(4+2 \mu) /\left(2+4 \mu+\mu^{2}\right)$ and $\lambda_{2}=\mu /((1+\mu)(2+\mu))$.

The proof can be carried out as in [20], [29], and [32]; we omit details. 


\section{REFERENCES}

1. J. Bear, Dynamics of Fluids in Porous Media, Dover, New York, 1972.

2. F. Brezzi, J. Douglas, Jr., R. Durán, and M. Fortin, Mixed finite elements for second order elliptic problems in three variables, Numer. Math. 51 (1987), 237-250.

3. F. Brezzi, J. Douglas, Jr., M. Fortin, and L. Marini, Efficient rectangular mixed finite elements in two and three space variables, RAIRO Modèl. Math. Anal. Numér 21 (1987), 581-604.

4. F. Brezzi, J. Douglas, Jr., and L. Marini, Two families of mixed finite elements for second order elliptic problems, Numer. Math. 47 (1985), 217-235.

5. M. Celia and P. Binning, Two-phase unsaturated flow: one dimensional simulation and air phase velocities, Water Resources Research 28 (1992), 2819-2828.

6. G. Chavent and J. Jaffré, Mathematical Models and Finite Elements for Reservoir Simulation, NorthHolland, Amsterdam, 1978.

7. Z. Chen, Analysis of mixed methods using conforming and nonconforming finite element methods, RAIRO Modèl. Math. Anal. Numér. 27 (1993), 9-34.

8. _ Finite element methods for the black oil model in petroleum reservoirs, IMA Preprint Series \# 1238, submitted to Math. Comp.

9. Z. Chen and J. Douglas, Jr., Approximation of coefficients in hybrid and mixed methods for nonlinear parabolic problems, Mat. Aplic. Comp. 10 (1991), 137-160.

10. _ Prismatic mixed finite elements for second order elliptic problems, Calcolo 26 (1989), 135148.

11. Z. Chen, R. Ewing, and M. Espedal, Multiphase flow simulation with various boundary conditions, Numerical Methods in Water Resources, Vol. 2, A. Peters, et als., eds., Kluwer Academic Publishers, Netherlands, 1994, pp. 925-932.

12. S. Chou and Q. Li, Mixed finite element methods for compressible miscible displacement in porous media, Math. Comp. 57 (1991), 507-527.

13. P. Ciarlet, The Finite Element Method for Elliptic Problems, North-Holland, Amsterdam, 1978.

14. J. Douglas, Jr., Finite difference methods for two-phase incompressible flow in porous media, SIAM J. Numer. Anal. 20 (1983), 681-696.

15. J. Douglas, Jr. and J. Roberts, Numerical methods for a model for compressible miscible displacement in porous media, Math. Comp. 41 (1983), 441-459.

16. __ Global estimates for mixed methods for second order elliptic problems, Math. Comp. 45 (1985), 39-52.

17. N. S. Espedal and R. E. Ewing, Characteristic Petrov-Galerkin subdomain methods for two phase immiscible flow, Comput. Methods Appl. Mech. Eng. 64 (1987), 113-135.

18. R. Ewing and M. Wheeler, Galerkin methods for miscible displacement problems with point sources and sinks-unit mobility ratio case, Mathematical Methods in Energy Research, K. I. Gross, ed., Society for Industrial and Applied Mathematics, Philadelphia, 1984, pp. 40-58.

19. K. Fadimba and R. Sharpley, A priori estimates and regularization for a class of porous medium equations, Preprint, submitted to Nonlinear World.

20. - Galerkin finite element method for a class of porous medium equations, Preprint.

21. D. Hillel, Fundamentals of Soil Physics, Academic Press, San Diego, California, 1980.

22. C. Johnson and V. Thomée, Error estimates for some mixed finite element methods for parabolic type problems, RAIRO Anal. Numér. 15 (1981), 41-78.

23. H. J. Morel-Seytoux, Two-phase flows in porous media, Advances in Hydroscience 9 (1973), 119-202.

24. J. C. Nedelec, Mixed finite elements in $\Re^{3}$, Numer. Math. 35 (1980), 315-341.

25. J. Nitsche, $L_{\infty}$-Convergence of Finite Element Approximation, Proc. Second Conference on Finite Elements, Rennes, France, 1975.

26. D. W. Peaceman, Fundamentals of Numerical Reservoir Simulation, Elsevier, New York, 1977.

27. O. Pironneau, On the transport-diffusion algorithm and its application to the Navier-Stokes equations, Numer. Math. 38 (1982), 309-332.

28. P.A. Raviart and J.M. Thomas, A mixed finite element method for second order elliptic problems, Lecture Notes in Math. 606, Springer, Berlin, 1977, pp. 292-315.

29. M. Rose, Numerical Methods for flow through porous media I, Math. Comp. 40 (1983), 437-467.

30. A. Schatz, V. Thomée, and L. Wahlbin, Maximum norm stability and error estimates in parabolic finite element equations, Comm. Pure Appl. Math. 33 (1980), 265-304. 
31. R. Scott, Optimal $L^{\infty}$ estimates for the finite element method on irregular meshes, Math. Comp. 30 (1976), 681-697.

32. D. Smylie, A near optimal order approximation to a class of two sided nonlinear degenerate parabolic partial differential equations, Ph. D. Thesis, University of Wyoming, 1989.

33. M. F. Wheeler, A priori $L_{2}$ error estimates for Galerkin approximation to parabolic partial differential equations, SIAM J. Numer. Anal. 10 (1973), 723-759.

Department of Mathematics and the Institute for Scientific Computation, Texas A\&M University, College Station, TX 77843.

E-mail address: zchen@isc.tamu.edu, ewing@ewing.tamu.edu

Department of Applied Mathematics, University of Bergen, Allegt. 55, N5007 Bergen, NORWAY.

E-mail address: Magne.Espedal@mi.uib.no 